Conclusions In a busy inner-city STD clinic, EPT is more likely to be accepted by women, those who are younger and those infected with $\mathrm{Ct}$. Re-infection rates among patients returning to the clinic suggest that EPT reduces the risk of re-infection, with the greatest benefit among those originally infected with GC.

\section{5-S3.02 IMPLEMENTATION OF AN EXPEDITED PARTNER THERAPY (EPT) PROGRAM IN AN INNER-CITY STD CLINIC}

doi:10.1136/sextrans-2011-050109.164

T Mickiewicz, A Al-Tayyib, C Mettenbrink, C Rietmeijer. Denver Public Health Denver, USA

Background Expedited partner therapy (EPT) is the practice of treating sex partners of persons with sexually transmitted diseases (STD) without an intervening medical evaluation. In 2006, the CDC issued guidelines for providing EPT to heterosexual patients diagnosed with gonorrhoea/chlamydial infection, allowing them to deliver treatment to their partner(s). In November 2006, the Denver Metro Health (STD) Clinic (DMHC) began offering EPT. We describe EPT implementation challenges and process improvements. Methods Three phases of implementation are described: 1) the pilot phase, 2) implementation of a quality assurance protocol and 3) initiation of a prompt in the electronic medical record (EMR). Data were extracted from the EMR to calculate acceptance rates of EPT among the 2056 eligible patients over this time period. Rates were examined by month and for each phase.

Results Before initiation of the pilot phase, a protocol to dispense EPT was developed and staff trained. During the pilot phase (September 2007-April 2008), patient acceptance rates averaged 17\% (range: $8 \%-22 \%$ ). As rates were not improving, EMR data were examined to determine potential areas for intervention. At DHMC, clinical services are provided by health care partners (HCP) and licensed nurse practitioners (LNP). Analyses revealed significant differences in patient acceptance of EPT by provider type: HCP providers were less likely to have patients accept EPT than LNP providers (prevalence ratio $=1.7,95 \% \mathrm{CI}: 1.5 \%$ to $1.9 \%$ ), likely due to the nature of the visit and the volume of patients seen by HCP providers. Targeted re-training was initiated and quality assurance reports were generated monthly. Although the acceptance rate increased significantly to $22 \%(p=0.04)$, there was no indication that rates were increasing with time. In January 2009, the EMR was amended, requiring all providers to document EPT referral, accept- ance or refusal (and reason for refusal), before closing the patient EMR. Rates improved significantly to an overall rate of $49 \%$ $(p<0.01)$ through October 2010 (Abstract O5-S3.02 figure 1) Reasons for refusal among those who did not accept EPT included that partner would be notified (42.5\%), partner was being treated $(30.1 \%)$ or the patient was not able to contact the partner $(21.9 \%)$ Conclusions In a busy inner-city STD clinic, an automated EMR prompt that forces documentation of EPT provided the greatest success. An EPT acceptance rate of 50\% may be the optimum rate in a real-world setting.

\section{5-S3.03 INCREMENTAL COST-EFFECTIVENESS OF INTRODUCING PARTNER NOTIFICATION WITH SELECTIVE SCREENING FOR STD CONTROL IN LOUISIANA}

doi:10.1136/sextrans-2011-050109.165

${ }^{1} \mathrm{M}$ Rahman, ${ }^{2} \mathrm{M}$ Khan, 'L Longfellow. 'Louisiana Office of Public Health, Metairie, USA; ${ }^{2}$ Tulane University, New Orleans, USA

Background Selective screening and partner notification are the two strategies used by the STD control programs in USA to reduce and or eliminate syphilis. So far no study has assessed the cost and effectiveness of either approach at the state level. The objective of this study is to assess the incremental cost effectiveness (ICE) of adding partner notification with selective screening (SS) in detecting early syphilis and to measure ICE of intensity of partner notification in Louisiana.

Method The cost effectiveness analysis (CEA) was done from the point of view of health care delivery. Micro costing approach was used in cost analysis and the CEA was performed by using the recurrent direct costs associated with detecting syphilis by SS and by SS with partner notification see Abstract O5-S3.03 figure 1. For ICE of intensity of partner notification, cost was calculated for every attempt made to contact a partner and effectiveness was calculated by the number of partners identified as well as the number of cases identified through partner notification.

Results The estimates of direct costs associated with SS was $\$ 6.4$ million for 1005 early syphilis cases detected and \$6.7 million for SS with partner notification. Partner notification detected additional 279 early syphilis cases with an additional cost of $\$ 314498$. Incremental cost of adding partner notification with SS was $\$ 2808$ per primary and secondary syphilis case, $\$ 1883$ per early latent syphilis

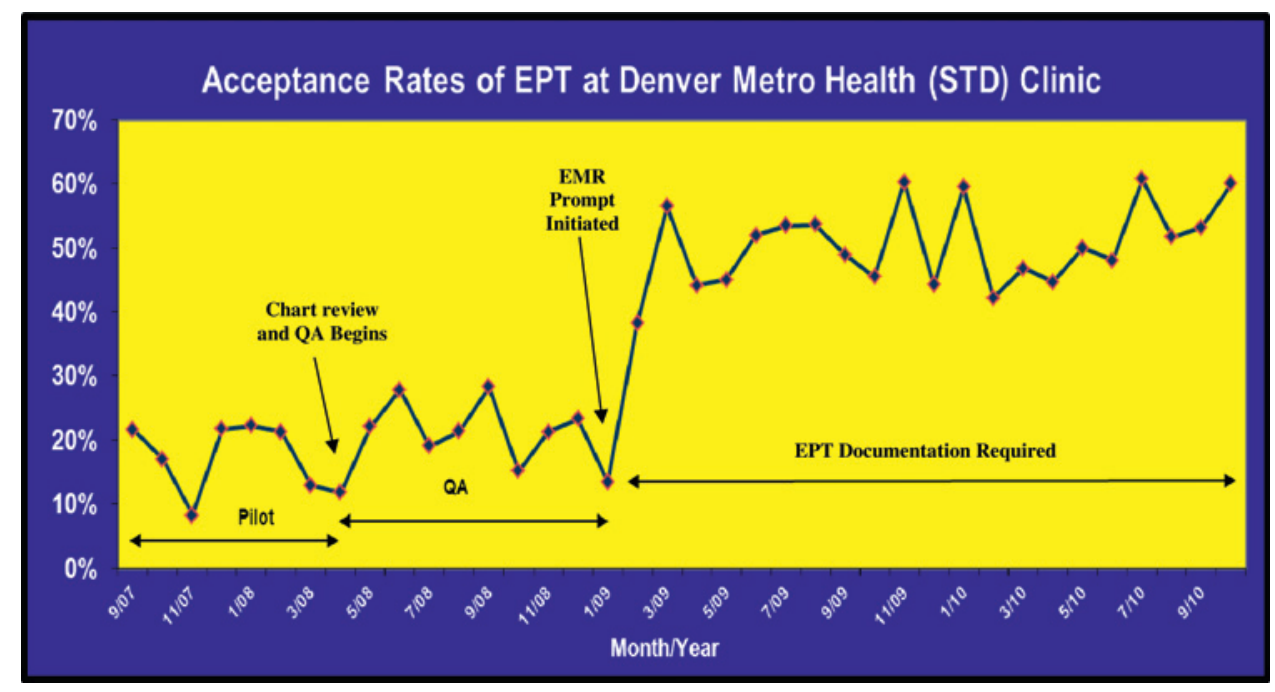

Abstract 05-S3.02 Figure 1 Acceptance rates of EPT Denver Metro Health (STD) clinic. 